\title{
The impact of serum lipids on risk for microangiopathy in patients with type 2 diabetes mellitus
}

Peter P Toth ${ }^{1,2^{*}}$, Robert J Simko ${ }^{3 \dagger}$, Swetha Rao Pallii ${ }^{4 \dagger}$, Dawn Koselleck ${ }^{3 \dagger}$, Ralph A Quimbo ${ }^{4 \dagger}$ and Mark J Cziraky ${ }^{4 \dagger}$

\begin{abstract}
Background: Few large-scale, real-world studies have assessed the relative associations of lipid fractions with diabetic microvascular events. The main objective of this study was to evaluate the association of the lipid profile components, high density lipoprotein cholesterol (HDL-C), low density lipoprotein cholesterol (LDL-C), triglycerides (TG), and non-high density lipoprotein cholesterol (non-HDL-C) with microvascular complications (MVCs) in type 2 diabetes mellitus (T2DM) patients.
\end{abstract}

Methods: This observational cohort study queried the HealthCore Integrated Research Database $\left(H I R D^{S M}\right)$ for newly-diagnosed (Index Date) 18-64-year-old patients with diabetes mellitus between 01/01/2005-06/30/2010. Inclusion required $\geq 12$ months pre-index continuous health plan eligibility and $\geq 1$ pre-index lipid profile result. Patients with polycystic ovary syndrome and prior MVCs were excluded. Incident complications were defined as the earliest occurrence of diabetic retinopathy, peripheral neuropathy, and/or nephropathy post-index. Cox proportional models and Kaplan-Meier (KM) curves were used to evaluate associations among variables.

Results: Of the patients ( $\mathrm{N}=72,267), 50.05 \%$ achieved HDL-C, $64.28 \%$ LDL-C, $59.82 \%$ TG, and $56.79 \%$ non-HDL-C American Diabetes Association goals at baseline. During follow-up (mean, 21.74 months), there were 5.21 microvascular events per 1,000 patient-months. A $1-\mathrm{mg} / \mathrm{dL}$ increase in $\mathrm{HDL}-\mathrm{C}$ was associated with $1 \%$ decrease in any MVC risk $(P<.0001)$, but for $\mathrm{LDL}-\mathrm{C}, \mathrm{TG}$, and non-HDL-C, 1-mg/dL increase resulted in increases of $0.2 \%$ $(P<.0001), 0.1 \%(P<0.001)$ and $0.3 \%(P<0.001)$ in MVC risk. Patients achieving HDL-C goals had a $11 \%$ lower risk of MVC versus non-achievers (RR 0.895, [95 \% Cl, 0.852-0.941], $P<.0001$ ). Similarly, TG goal attainment was associated with a lowered risk for any MVC (RR 0.849, [95 \% Cl, 0.808-0.892], $P<.0001$ ). Evaluation of KM survival curves demonstrated no significant difference in the risk of MVCs between patients achieving vs. not achieving LDL-C goals, but did demonstrate a difference in MVC risk between patients achieving vs. not achieving non-HDL-C goals.

Conclusion: This study demonstrates significant independent associations among lipid fractions and risk for microangiopathy. These findings suggest that attaining established ADA goals for HDL-C, TG, and non-HDL-C may reduce risk for microvascular events among patients with diabetes.

Keywords: Lipid subfractions, ADA treatment goals, Diabetes, Microvascular complications, Retinopathy, Neuropathy, Nephropathy

\footnotetext{
* Correspondence: Peter.Toth@cghmc.com

${ }^{\dagger}$ Equal contributors

${ }^{1}$ CGH Medical Center, 101 east Miller Rd., Sterling, IL 61081, USA

${ }^{2}$ University of Illinois College of Medicine, Peoria, IL, USA

Full list of author information is available at the end of the article
} 


\section{Background}

An estimated 25.8 million people, or $8.3 \%$ of the US population, were affected by diabetes mellitus in 2010, with $90 \%-95 \%$ afflicted with type 2 (T2DM). The American Diabetes Association (ADA) and American Heart Association (AHA) attribute substantial patient morbidity and mortality to T2DM [1]. Furthermore, T2DM is associated with multiple microvascular complications (MVCs) including retinopathy, neuropathy, and nephropathy, all of which contribute to diabetesassociated morbidity and mortality [2,3]. From 20052008, 4.2 million patients with diabetes had diabetic retinopathy, 655,000 of whom developed serious vision loss [4] for a total annual cost of $\$ 492.98$ million [5]. Diabetic neuropathy affects an estimated $12 \%-50 \%$ of those with diabetes, with total annual costs estimated at $\$ 10.1$ billion. As many as $19 \%$ of T2DM patients have nephropathy and end-stage renal disease, with annual medical costs in the US amounting to about $\$ 15$ billion [6].

Elevated serum levels of low-density lipoprotein cholesterol (LDL-C) and triglycerides (TG) and low levels of high-density lipoprotein cholesterol (HDL-C) are strongly associated with increased risk for macrovascular events (e.g., myocardial infarction, ischemic stroke, and coronary mortality) among patients with T2DM [3,7,8]. However, no consensus exists on possible mechanisms linking these individual lipid subfractions to MVCs. A systematic review of studies assessing associations between lipid subfractions and MVCs noted that dyslipidemia may cause or worsen complications [3]. A European study reported significant associations between elevated levels of total cholesterol (TC) and lower levels of HDL-C with increasing severity of diabetic retinopathy; there was, however, no apparent association between serum TG and retinopathy [9]. Another study found high levels of TC and TG associated with diabetic nephropathy and declining kidney function [10]. The relationship between abnormal lipid subfractions and diabetic neuropathy is relatively unexplored, with only one study showing a small degree of association [11]. Most studies seeking to establish associations between dyslipidemia and microvascular events were of short duration with relatively small sample sizes, rendering them underpowered and with limited generalizability [3]. Nevertheless, available evidence indicates abnormalities among lipid subfractions exacerbate the risk of microvascular events [9].

To date, few studies have investigated the relative magnitude of association between individual lipid subfractions and diabetes-related MVCs. Furthermore, comprehensive literature reviews have not revealed any large-scale realworld studies that assessed the relative associations of standard lipid fractions with diabetic MVCs. In recognition of that gap, this study accessed data from a managed care database to evaluate associations between the levels of lipid subfractions (LDL-C, HDL-C, TG, and non-HDL-C) and the incidence of diabetic retinopathy, peripheral neuropathy, and nephropathy. The impact on risk for MVCs following attainment of ADA targets for specific components of the lipid profile was also quantified [12].

\section{Methods}

\section{Data source and study design}

This observational cohort study utilized integrated medical, pharmacy, and laboratory result data from the HealthCore Integrated Research Database ${ }^{\mathrm{SM}}\left(\mathrm{HIRD}^{\mathrm{SM}}\right)$ between 01/01/04-06/30/2010. The HIRD ${ }^{\text {SM }}$ contains administrative claims data for more than 35 million Americans covered by 14 geographically dispersed commercial US healthcare plans. All information utilized in this retrospective, longitudinal analysis was managed in strict compliance with the Health Insurance Portability and Accountability Act (HIPAA) of 1996. Patient confidentiality and anonymity was preserved via de-identification throughout the study.

\section{Inclusion criteria}

Patients newly diagnosed with T2DM (index date) involving a medical claim with an International Classification of Diseases, Ninth Revision, Clinical Modification (ICD9-CM) diagnosis code for $250 . x 0$ or $250 . x 2$ or a pharmacy claim with a GPI for an antidiabetic agent between 01/01/ 2005 and 06/30/2010 were identified. The index date was the earliest point of onset of T2DM. To be included, patients aged between 18-to-64 years were required to have $\geq 12$ months of continuous health plan eligibility preceding the index date and $\geq 1$ full lipid panel during the 12 -month pre-index date period, the most current of which was defined as the baseline lipid panel.

\section{Exclusion criteria}

Patients with a history of T2DM or any pre-index medical claim for diabetic retinopathy, neuropathy, or reduced kidney function were excluded. Also excluded were patients with a medical claim for polycystic ovary syndrome (ICD-9-CM: 256.4x) any time during the study period.

\section{Definition of follow-up}

Follow-up was defined as the period between the index date and the earliest of the following: end of continuous health plan eligibility, end of the available data stream $(06 / 30 / 2010)$, or a censoring event. For each outcome of interest, data were censored at the earliest occurrence of the event of interest, when patients reached 65 years of age, or a death was recorded in the Social Security Death Index Master File. Adjustments in the amount of persontime contributed during follow-up were made whenever 
applicable to account for follow-up periods of varying durations.

\section{MVCs}

The MVCs of interest were diabetic neuropathy, retinopathy, and nephropathy identified during any inpatient, outpatient, or emergency room visit using ICD-9-CM diagnoses and procedure codes, Current Procedural Terminology $(\mathrm{CPT})$ codes, and revenue service codes from patients' medical claims. Nonspecific diagnoses of neuropathy and nephropathy were considered to be T2DMattributable MVCs provided a T2DM diagnosis code was recorded on the same medical claim date. In addition, diabetic nephropathy was also identified using glomerular filtration rates (eGFR) estimated from the serum creatinine laboratory values. An abbreviated Modification of Diet and Renal Disease (MDRD) equation was used to calculate eGFR since information on race was lacking [13]. An eGFR of $<60 \mathrm{~mL} / \mathrm{min}$ was defined as reduced kidney function, although this may not necessarily be attributable to diabetes. As a result, any eGFR-identified overt nephropathy was only classified as diabetic nephropathy if there was no prior medical claim for nonspecific nephropathy.

\section{Lipid subfraction goals}

Results from the last full lipid panel measurement before the end of the follow-up period was used to evaluate the relative association between each lipid subfraction and the events of interest. The target level for each lipid fraction was defined on the basis of ADA treatment guidelines for people with diabetes: LDL-C $<100 \mathrm{mg} / \mathrm{dL}$, HDL-C $>40 \mathrm{mg} / \mathrm{dL}$ for men and $>50 \mathrm{mg} / \mathrm{dL}$ for women, TG $<150 \mathrm{mg} / \mathrm{dL}$, and non-HDL-C $<130 \mathrm{mg} / \mathrm{dL}$ [12]. Assessment of lipid goals as of the baseline lipid panel (i.e., preceding the onset of T2DM), were applied according to the National Cholesterol Education Program Adult Treatment Panel III (NCEP ATP III) Guidelines for nondiabetic patients [13]. Depending on their risk category primary (2+ risk factors), secondary (cardiovascular heart disease [CHD] or CHD risk equivalents), or nondeterminant ( $0-1$ risk factors) based on information available from their start date of continuous eligibility until the day of the lipid panel measurement - the following rules for goal attainment were applied: LDL-C $<130 \mathrm{mg} / \mathrm{dL}$ and $<100 \mathrm{mg} / \mathrm{dL}$ for patients at primary and secondary risk, respectively; HDL-C $>40 \mathrm{mg} / \mathrm{dL}$ for men and $>50 \mathrm{mg} / \mathrm{dL}$ for women; TG $<150 \mathrm{mg} / \mathrm{dL}$ for women (based on AHA guidelines for women [14]) and $<200 \mathrm{mg} / \mathrm{dL}$ for men; and non-HDL-C $<160 \mathrm{mg} / \mathrm{dL}$ and $<130 \mathrm{mg} / \mathrm{dL}$ for patients at primary and secondary risk, respectively. For patients with an indeterminate risk status, baseline LDL-C and nonHDL-C goal attainment could not be ascertained.

\section{Patient characteristics}

Demographic characteristics included age, gender, geographic location, and type of health insurance coverage on index date. Baseline comorbidities and the DeyoCharlson Comorbidity Index were evaluated during the 12 -month pre-index period. The most recent lipid subfractions of interest (LDL-C, HDL-C, TG, and nonHDL-C) prior to the index date were used to describe the baseline lipid panel.

Medication utilization patterns were assessed for antiglycemic, antihypertensive, and lipid-altering therapies at baseline and follow-up. Exposure to both antidiabetic and antihypertensive agents (angiotensin-converting enzyme [ACE] inhibitors and angiotensin receptor blockers [ARBs]) during follow-up was measured based on the proportion of days covered (PDC) $[15,16]$. Exposure to lipidaltering therapies, such as statins, niacin, and fibrates (fenofibrate and gemfibrozil), as of the latest available full lipid laboratory panel prior to the end of follow-up. Was evaluated to adjust for potential changes in lipid values between the last observed lipid panel and the end of follow-up.

\section{Statistical analyses \\ Descriptive statistics}

Continuous characteristics were represented using means and standard deviations, while categorical variables were evaluated as percentages. All right-censored continuous outcomes were compared by means of unadjusted Cox proportional hazards models and the log rank test. Kaplan-Meier (KM) curves were constructed to evaluate the risk of MVCs between patients achieving and not achieving goals for each lipid subfraction.

\section{Multivariate analysis}

Four Cox proportional hazards models were developed to determine the relative significance of lipid subfraction levels and goal attainment on the risk of MVCs. Additional covariates included age, gender, state of residence, health plan, and physician type. Also included were preindex health conditions (hypertension, obesity, heart failure, metabolic syndrome, renal disease, liver disease, ischemic heart disease, peripheral vascular disease, and depression) and PDC during follow-up for antihypertensives (ACEs and ARBs) and lipid-altering therapies (statins, niacin, ezetimibe, and fibrates).

\section{Sensitivity analysis}

Additional univariate assessments were performed to determine if the observed associations between TG/HDL and MVCs remained unchanged or attenuated once TG was adjusted for HDL-C or vice versa by computing the variance inflation factor (VIF) and condition index. A value of 10 and 30 respectively were set apriori to 
indicate a potential issue of multicollinearity. In order to further investigate the relative importance of the management of other lipid measures beyond LDL-C alone with respect to MVC risk, the risk of MVCs among patients achieving LDL-C goals only vs. those simultaneously achieving HDL-C and LDL-C goals was evaluated. Similar comparisons were conducted between those achieving LDL-C goal only vs. those simultaneously achieving dual goals of LDL-C and TG, HDL-C and TG and non-HDL-C goals respectively. Furthermore, the above analyses were replicated among those who did not attain LDL-C treatment goals to evaluate the impact of HDL-C, TG, and Non-HDL-C goal achievement in the absence of LDL-C goal achievement.

\section{Results}

\section{Demographic and clinical characteristics at baseline}

Of the patients $(\mathrm{N}=72,267)$ in this study, $48.7 \%$ were females, and the overall mean $( \pm$ SD) age was 49.91 $( \pm 9.44)$ years (Table 1$)$. At baseline, the mean $( \pm$ SD) lipid panel values for all patients were $115.71( \pm 35.65)$ for LDL-C, $47.28( \pm 13.90)$ for HDL-C, $186.69( \pm 176.52)$ for TG, and $150.61( \pm 43.53)$ for non-HDL-C. Overall, $50.05 \%$ of the patients attained their HDL-C goals, while $59.82 \%$ reached their TG goals at baseline. Based on a subsample of 37,118 patients for whom CHD risk status and corresponding LDL-C and non-HDL-C goals could be determined, $64.28 \%$ of the patients attained their LDL-C goals, while $56.79 \%$ reached their nonHDL-C goals at baseline.

\section{Follow-up medication utilization}

Assessment of medication utilization during follow-up revealed no significant differences between any medication class (i.e., antiglycemic, antihypertensive, and lipidaltering drugs) irrespective of whether patients had or did not have an MVC event for any of the lipid subfractions.

\section{Follow-up MVC incidence rate}

On the basis of 1000 patient-months, the incidence rate for any microvascular event was $5.21(95 \% \mathrm{CI}, 5.09$ 5.33). Incidence rates were 1.44 ( $95 \% \mathrm{CI}, 1.38-1.50)$ for diabetic retinopathy; 1.26 (95\% CI, 1.20-1.32) for diabetic neuropathy; and 2.61 (95\% CI, 2.53-2.69) for diabetic nephropathy.

\section{Follow-up Lipid Subfractions}

As of the last full lipid panel measurement during follow-up, the mean HDL-C, TG, and non-HDL-C values for patients who experienced any MVC event were significantly different compared with patients with no events $(P<.0001$; Table 2$)$. The mean HDL-C values in the total population was $48.02( \pm 14.13)$; for patients with an observed MVC event, the mean HDL-C was 46.72 $( \pm 14.04)$; and for patients without events, $48.16( \pm 14.13$, $P<.0001)$. The mean LDL-C values for patients who experienced MVC events versus those with no event were not significantly different.

LDL-C goal attainment occurred more frequently among patients with events (43.8\%) versus those who had no event (41.86 \%, $P=.0015)$. Patients who experienced an MVC event were less likely to have attained their HDL-C and TG goals ( $48.51 \%$ and $54.30 \%$, respectively) compared with patients with no event (52.96\% and $60.97 \%$, for HDL-C and TG, respectively, $P<.001)$. There was no significant difference in the proportion of patients achieving non-HDL-C goals between patients with MVC events $(41.70 \%)$ compared with those with no events $(42.63 \%, P=.1299)$.

\section{Univariate and multivariate comparisons between lipid subfractions and MVCs}

MVC incidence rates per 1000 patient-months were evaluated for each lipid subfraction based on the laboratory values from the last tests before the first event or censorship.

\section{LDL-C}

The MVC incidence rate for patients who attained their LDL-C goals was 5.17 per 1000 patient-months versus 5.24 for those who did not attain their LDL-C goals during follow up. A unit increase in LDL-C was not associated with a significant risk increase (RR 1.0, [95 \% CI, $1-1.001], P=.3513)$. KM curves showed no significant difference in risk for any MVC event and LDL-C goal attainment (RR 1.011, [95 \% CI, 0.965-1.059], $P=.6522$; Figure 1). Multivariate Cox regression analysis, however, demonstrated significant associations between LDL-C levels (RR 1.002, [95 \% CI, 1.001-1.002], $P<.0001$ ) and LDL-C goal attainment (RR 0.909, [95 \% CI, 0.8650.955], $P=.0001$ ) and MVC risk (Table 3).

\section{HDL-C}

The MVC incidence rate was 4.73 per 1000 patientmonths for patients at their HDL-C goal versus 5.76 per 1000 patient-months who were not. Each unit increase in HDL-C was associated with $1 \%$ decrease in event risk (RR 0.992, [95 \% CI, 0.99-0.994], $P<.0001)$. Patients who attained HDL-C goals had $17 \%$ lower risk of any MVC event versus those who did not (RR 0.83, [95\% CI, 0.793$0.869], P<.0001$; Figure 1 ). Adjusted analysis showed that a one unit increase in HDL-C levels or HDL-C goal attainment reduced MVC risk by $0.5 \%$ (RR 0.995, [95\% CI, 0.993-0.997]; $P<.0001$ ) and $10.5 \%$ (RR 0.895, [95\% CI, 0.852-0.941]; $P<.0001)$, respectively. 
Table 1 Baseline Characteristics of Patients in the Study

\begin{tabular}{|c|c|c|}
\hline \multirow[t]{2}{*}{ Characteristics } & \multicolumn{2}{|c|}{$\begin{array}{c}\text { Patients } \\
\mathrm{N}=72,267\end{array}$} \\
\hline & $\mathrm{n}$ & $\%$ \\
\hline Female & 35,192 & 48.70 \\
\hline Age at index (years), mean $\pm S D$ & 49.91 & \pm 9.44 \\
\hline \multicolumn{3}{|l|}{ Geographic Region } \\
\hline West & 21,098 & 29.19 \\
\hline South & 19,727 & 27.30 \\
\hline Northeast & 15,756 & 21.80 \\
\hline Midwest & 11,231 & 15.54 \\
\hline Unknown & 4,455 & 6.16 \\
\hline \multicolumn{3}{|l|}{ Health Plan Type } \\
\hline $\mathrm{HMO}$ & 35,017 & 48.46 \\
\hline PPO & 31,556 & 43.67 \\
\hline POS & 2,347 & 3.29 \\
\hline FFS & 65 & 0.09 \\
\hline Other & 3,255 & 4.50 \\
\hline \multicolumn{3}{|l|}{ Index year } \\
\hline 2005-06 & 21,929 & 30.34 \\
\hline 2007-08 & 30,767 & 42.57 \\
\hline 2009-10 & 19,571 & 27.08 \\
\hline \multicolumn{3}{|l|}{ Physician specialty } \\
\hline General family practice & 22,927 & 31.34 \\
\hline Internal medicine & 17,817 & 24.65 \\
\hline Endocrinology & 1,963 & 2.72 \\
\hline Cardiology & 800 & 1.11 \\
\hline Others/unknown & 28,760 & 39.80 \\
\hline \multicolumn{3}{|l|}{ Lipid Subfraction ${ }^{1}$} \\
\hline LDL-C & 115.71 & \pm 35.65 \\
\hline Goal attainment ${ }^{2}$ & 23,858 & 64.28 \\
\hline $\mathrm{HDL}-\mathrm{C}$ & 47.28 & \pm 13.90 \\
\hline Goal attainment & 36,171 & 50.05 \\
\hline TG & 186.69 & \pm 176.52 \\
\hline Goal attainment & 43,230 & 59.82 \\
\hline nonHDL-C & 150.61 & \pm 43.53 \\
\hline Goal attainment ${ }^{2}$ & 21,081 & 56.79 \\
\hline
\end{tabular}

\section{Comorbidities}

Ischemic heart disease

Hypertension

Obesity

Depression

Heart failure

Cerebrovascular disease

Metabolic syndrome

Liver disease
Table 1 Baseline Characteristics of Patients in the Study (Continued)

\begin{tabular}{lcc}
\hline Peripheral vascular disease & 1,554 & 2.15 \\
Renal disease $^{3}$ & 394 & 0.55 \\
Schizophrenia & 68 & 0.09 \\
DCl score, mean \pm SD & 0.34 & \pm 0.9 \\
\multicolumn{1}{c}{ Medication Use } & & \\
Antihypertensive Medications & & 19.91 \\
ACE Inhibitors & 14,387 & 14.45 \\
ARBs & 10,444 & 6.25
\end{tabular}

\section{Lipid Altering Therapies}

\begin{tabular}{|c|c|c|}
\hline Low Potency & 2,422 & 3.35 \\
\hline Medium Potency & 9,840 & 13.62 \\
\hline High Potency & 12,851 & 17.78 \\
\hline Niacin & 1,668 & 2.31 \\
\hline Ezetimibe & 3,827 & 5.30 \\
\hline \multicolumn{3}{|l|}{ Fibrate } \\
\hline Fenofibrate & 3,211 & \\
\hline Gemfibrozil & 1,254 & 1.74 \\
\hline Days of Study Follow-up ${ }^{5}$, mean \pm SD & 652.58 & \pm 48 \\
\hline
\end{tabular}

${ }^{1}$ The lipid panel values are derived from the most recent lab assessment results prior to the index date.

${ }^{2}$ For LDL-C and non-HDL-C: Goal attainment based on subsample of 37,118 patients since 35,149 patients had no discernible risk at baseline.

${ }^{3}$ Pre-index renal disease includes acute renal failure, hypertensive kidney disease, and glomerulonephritis.

${ }^{4} \mathrm{n}$ and $\%$ of patients with any fill for medication of interest.

${ }^{5}$ Number of days from index date to end of study follow up (defined as the end of earliest occurrence of event of interest, death, patient becoming 65 years or older, end of health plan eligibility, or 6/30/2010).

LDL-C: low-density lipoprotein cholesterol; HDL-C: high-density lipoprotein cholesterol; TG: triglycerides; ACE: angiotensin-converting enzyme; ARB: angiotensin receptor blockers; HMO: health maintenance organization; PPO: patient preferred organization; POS: point of service; FFS: fee for service.

\section{TG}

Patients achieving TG goal had an MVC incidence rate of 4.71 per 1000 patient-months versus those who did not (5.97). A unit increase in TG levels resulted in a $0.1 \%$ greater risk of a MVC event (RR 1.001, [95 \% CI, 1.001-1.001], $P<.0001)$. Patients at TG goal were $11.3 \%$ less likely to have a microvascular complication versus those not attaining target TG levels (RR 0.787, [95\% CI, 0.751-0.824], $P<.0001$; Figure 1). Multivariate regression analysis also indicated significant associations between TG levels (RR 1.001, [95 \% CI, 1.001-1.001], $P<.0001$ ) and TG goal achievement (RR 0.849, [95\% CI, 0.8080.892], $P<.0001)$ and MVC risk.

\section{Non-HDL-C}

For patients achieving non-HDL-C goal, the MVC incidence rate was 4.83 versus 5.52 per 1000 patient-months for those not at goal. For each unit increase in non- 
Table 2 Last Lipid Panel Results Prior to Any Microvascular Event or Censorship

\begin{tabular}{|c|c|c|c|c|c|c|c|}
\hline \multirow{2}{*}{$\begin{array}{l}\text { Lipid Characteristics } \\
\text { \# Days from Last Lipid Panel to Any } \\
\text { Microvascular Event/Censorship: mean } \pm \text { SD }\end{array}$} & \multicolumn{2}{|c|}{$\begin{array}{l}\text { All Patients } \\
\mathrm{N}=72,267\end{array}$} & \multicolumn{2}{|c|}{$\begin{array}{c}\text { Event }^{1} \\
\mathrm{n}=7,271\end{array}$} & \multicolumn{2}{|c|}{$\begin{array}{l}\text { No Event }{ }^{2} \\
n=64,996\end{array}$} & \multirow{2}{*}{$\begin{array}{c}P \text { - Value } \\
<.0001\end{array}$} \\
\hline & 346.21 & \pm 351.43 & 148.40 & \pm 223.94 & 368.34 & \pm 356.15 & \\
\hline \multicolumn{8}{|l|}{ Lipid Panel Values: mean \pm SD } \\
\hline LDL-C & 109.08 & \pm 34.35 & 108.33 & \pm 36.47 & 109.16 & \pm 34.1 & .0513 \\
\hline $\mathrm{HDL}-\mathrm{C}$ & 48.02 & \pm 14.13 & 46.72 & \pm 14.04 & 48.16 & \pm 14.13 & $<.0001$ \\
\hline Triglycerides & 163.35 & \pm 122 & 186.39 & \pm 186.41 & 160.77 & \pm 112.23 & $<.0001$ \\
\hline Non-HDL-C & 140.55 & \pm 40.25 & 143.19 & \pm 45.9 & 140.26 & \pm 39.56 & $<.0001$ \\
\hline \multicolumn{8}{|l|}{ Lipid Goal Attainment Results: n, row\% } \\
\hline LDL-C & 30,395 & $42.06 \%$ & 3,185 & $43.80 \%$ & 27,210 & $41.86 \%$ & .0015 \\
\hline $\mathrm{HDL}-\mathrm{C}$ & 37,946 & $52.51 \%$ & 3,527 & $48.51 \%$ & 34,419 & $52.96 \%$ & $<.0001$ \\
\hline Triglycerides & 43,577 & $60.30 \%$ & 3,948 & $54.30 \%$ & 39,629 & $60.97 \%$ & $<.0001$ \\
\hline Non-HDL-C & 30,737 & $42.53 \%$ & 3,032 & $41.70 \%$ & 27,705 & $42.63 \%$ & .1299 \\
\hline
\end{tabular}

${ }^{1}$ For those patients who experienced any corresponding microvascular event of interest (diabetic retinopathy/nephropathy/neuropathy), mean values of the last laboratory assessment prior to the first event are presented.

${ }^{2}$ For those patients who did not experience the microvascular event of interest (diabetic retinopathy/nephropathy/neuropathy), mean values of the last laboratory assessment prior to censorship (death, age $>64$ years, end of plan eligibility, or data period) are presented.

${ }^{3} P$-value between the event and no event groups is significant at $<.05$.

LDL-C: low-density lipoprotein cholesterol; HDL-C: high-density lipoprotein cholesterol; TG: triglycerides.

HDL-C, the increase in MVC event risk was $0.3 \%$ (RR 1.003, [95 \% CI, 1.002-1.003], $P<.0001)$. Patients who attained non-HDL-C goal had $10 \%$ lower risk for a microvascular event compared with patients who did not (RR 0.90, [95 \% CI, 0.859-0.943], $P<.0001$; Figure 1). Adjusted Cox results indicated that non-HDL-C levels (RR 1.003, [95\% CI, 1.003-1.004], $P<.0001)$ and non-HDL-C goal (RR 0.827, [95 \% CI, 0.788-0.868], $P<.0001$ ) were significantly associated with lowered MVC event risks.

\section{Sensitivity analysis}

The reported associations for TG and HDL lipid subfractions remained free from any collinearity bias based on the minor $(<2)$ VIF and condition index values observed for the lipid-subfractions in question. This indicates that the associations between TG/HDL and MVCs were not affected by the presence of other lipid fractions in the same model. Irrespective of LDL-C treatment goal achievement, significant independent reductions in MVC risk were found for those who attained HDL-C, TG, HDL-C and TG and non-HDL-C goals versus those who did not achieve these goals (Table 4).

\section{Discussion}

Consistent with the available evidence on diabetic complications $[2,3,7,9-11,17,18]$, this study found significant independent associations between HDL-C, LDL-C, TG, and non-HDL-C and risks for MVC among patients with T2DM in a real-world managed care population. Although management of LDL-C is the primary goal of therapy, our findings suggest further benefit may be obtained by expanding treatment goals to include modification of other lipid subfractions in addition to LDL-C. These associations persisted after controlling for numerous covariates, including comorbidities and concomitant medications.

A $1 \mathrm{mg} / \mathrm{dL}$ increase in HDL-C levels was associated with a significant decrease $(0.5 \%)$ in the risk of MVCs. Furthermore, patients who achieved HDL-C goals during follow-up also reduced their risk for experiencing MVCs by $10.5 \%$. These findings suggest that targeting HDL-C in addition to LDL-C as recommended by NCEP ATP III may have benefits beyond CHD risk reduction [12]. In the case of TGs, a significant increase in risk (0.1\%) for a MVC event was observed for a $1 \mathrm{mg} / \mathrm{dL}$ increase in the serum levels of TG. Further underscoring the risks associated with suboptimal TG goals $[2,13,19,20]$, the study found TG goal attainment lowered the risk of MVC by $15.1 \%$. The relationship of elevated TG and/or reduced HDL-C levels with MVCs was recently demonstrated by Zoppini et al., who showed a high TG/HDL-C ratio approximately doubled the risk for MVC over a 5-year period in 979 Caucasian patients with T2DM [21]. In a prospective observational study of 6,499 patients with diabetes and dyslipidemia, Teramoto et al. confirmed the importance of controlling TG and HDL-C in conjunction with HbA1C levels in patients with T2DM [22]. Taken together, these studies highlight the importance of targeting atherogenic dyslipidemia as a means to reduce MVCs in patients with T2DM. Our results further extend these findings by emphasizing the need to treat HDL-C and triglycerides to ADA defined targets in order to beneficially impact risk for both macrovascular and microvascular events. 


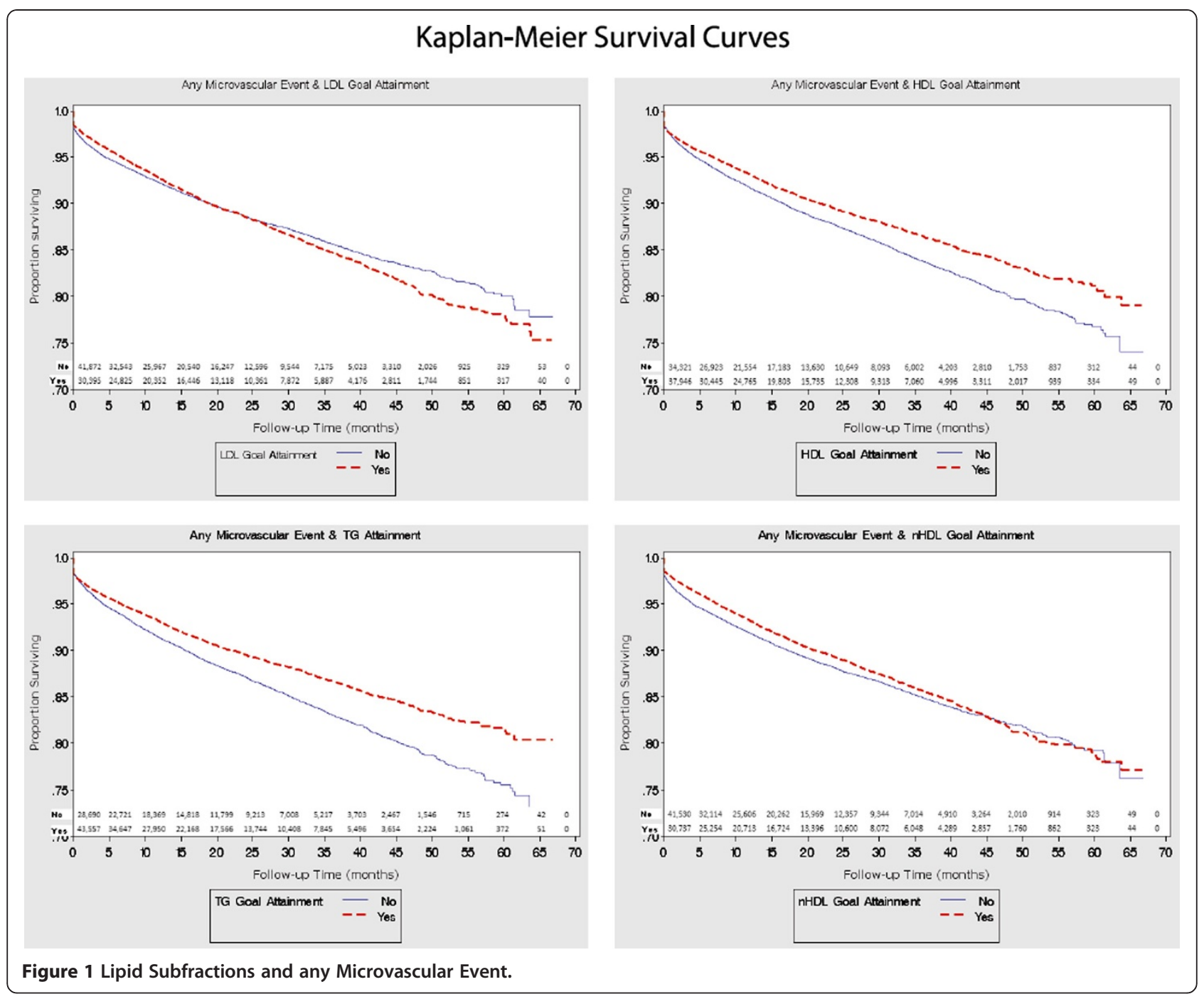

Continuous LDL-C levels and achievement of LDL-C goals were positively and negatively associated with MVC event risk, respectively in our study. However, the KM curves between those achieving versus those not achieving LDL-C goals were not statistically different $(P=.3513)$, indicating a lack of proportionality of MVC event risk over time. LDL-C reduction does not appear to mitigate risk for MVCs in diabetic patients.

Guidelines recommend non-HDL-C as a secondary target for therapy after risk-stratified LDL-C levels have been reached in patients with baseline TG $>200 \mathrm{mg} / \mathrm{dL}$ [23]. One of the key results in this study was the $17.3 \%$ reduction in the risk of MVCs for those attaining nonHDL-C goals compared to those not reaching the goals. Non-HDL-C, defined as TC minus HDL-C, is a sensitive surrogate measure of total atherogenic lipoprotein burden in serum and is a useful index for predicting the risk of CVD, especially among patients with diabetes since it circumvents the reliability issues associated with using
LDL-C in a diabetic population [24]. These findings hint at a potential role for non-HDL-C in predicting risk for microangiopathy in T2DM patients since it singularly combines the impact of total cholesterol and its various atherogenic lipoproteins in place of LDL-C. Furthermore, evidence exists that after achieving LDL-C goal levels, non-attainment of non-HDL-C target goals among T2DM patients is markedly associated with residual risk and dyslipidemia [25].

Overall, the current study highlights the possible benefits of treating any or all components of the lipid beyond LDL-C alone, in order to most optimally reduce morbidity and mortality in diabetic patients. The sensitivity analyses further confirmed the hypothesis that independently achieving HDL-C, TG, HDL-C and TG and non-HDL-C goals can lead to significant reductions in MVC risk irrespective of LDL-C goal attainment. This suggests that attainment of lipid goals other than LDL-C can translate into incremental reductions in risk of MVCs. 
Table 3 Cox Proportional Hazard Models: HDL-C, LDL-C, TG and non-HDL-C and the First Microvascular Event

\begin{tabular}{|c|c|c|c|c|c|c|}
\hline \multirow[t]{2}{*}{ Primary Risk Factors of Interest $^{1}$} & \multicolumn{3}{|c|}{ Lipid Panel $^{2}$} & \multicolumn{3}{|c|}{ Lipid Goal Attainment $^{2}$} \\
\hline & $\mathrm{HR}^{3}$ & 95 \% Cl & $P$-value & $\mathrm{HR}^{3}$ & $95 \%$ Cl & $P$-value \\
\hline HDL-C & 0.995 & $0.993-0.997$ & $<.0001$ & 0.895 & $0.852-0.941$ & $<.0001$ \\
\hline LDL-C & 1.002 & $1.001-1.002$ & $<.0001$ & 0.909 & $0.865-0.955$ & .0001 \\
\hline TG & 1.001 & $1.001-1.001$ & $<.0001$ & 0.849 & $0.808-0.892$ & $<.0001$ \\
\hline non-HDL-C & 1.003 & $1.003-1.004$ & $<.0001$ & 0.8270 & $0.788-0.868$ & $<.0001$ \\
\hline
\end{tabular}

${ }_{1}^{1}$ Four Cox proportional hazard models were run, two including HDL-C, LDL-C, and TG (lipid values and their goal attainment); the others with non-HDL-C lipid values and goal attainment as the primary risk factor of interest.

${ }^{2}$ The lipid panel values are derived from the last lab assessment results prior to the first microvascular (either retinopathy/nephropathy/neuropathy) event or censorship.

${ }^{3}$ The HRs were obtained after controlling for the demographics (age, gender and state of residence), other index characteristics (health plan and physician type), preindex health conditions (hypertension, obesity, heart failure, metabolic syndrome, renal disease, liver disease, ischemic heart disease, peripheral vascular disease, and depression), proportion of days covered during the follow up by the antihypertensives (ACEs, ARBs), and proportion of days covered by lipid altering therapies (statins [low, medium or high], niacin, ezetimibe, or fibrates [fenofibrates or genfibrozil]) between the last lipid panel measurement and event/censorship. HR: hazard ratio; Cl: confidence interval; LDL-C: low-density lipoprotein cholesterol; HDL-C: high-density lipoprotein cholesterol; TG: triglycerides.

Furthermore, this study lays the groundwork for conducting additional research to identify the best predictive models for estimating MVC risk (e.g., correlating Log (TG)/ HDL-C with risk for MVC) [26]. More importantly, these study findings suggest that a gap exists between the current practice recommendations and the appropriate management of MVC risk among T2DM patients.

Post hoc analyses of multiple clinical trials suggest that fenofibrate therapy impacts risk for MVC. The Fenofibrate Intervention and Event Lowering in Diabetes (FIELD) study found that patients receiving fenofibrate treatment had a reduced risk of a first and follow-up laser intervention for proliferative retinopathy, lower extremity amputation, and progression to albuminaria and nephropathy versus those on placebo [27-29]. Similarly, the Action to Control Cardiovascular Risk in Diabetes (ACCORD) group of studies evaluating the effect of intensive glycemic control and combination therapy for dyslipidemia found significantly reduced progression rates of diabetic retinopathy for patients who received fenofibrate versus those who received placebo [30]. The Diabetes Atherosclerosis Intervention Study (DAIS) reported reduced progression of albumin excretion for fenofibrate treatment versus placebo [31]. While the confounding effect arising out of fenofibrate usage was controlled for in the present analysis, our results underscore the relative importance of the other components of the standard lipid profile besides LDL-C to microangiopathy risk. They also emphasize the importance of appropriately managing HDL-C, TG, and,
non-HDL-C levels through therapeutic lifestyle changes and appropriate pharmacotherapy.

With the increasing prevalence of T2DM adding to the clinical and overall healthcare burdens of patients and society, there is an urgent need for optimal and timely disease management. In the absence of guidelines for the management of lipid subfraction levels to reduce the risk of diabetic microangiopathy, the control of these modifiable risk factors may lead to reducing or delaying the incidence of T2DM-related MVCs [29]. These findings suggest that there may be a need to simultaneously target improvements in multiple lipid subfractions beyond LDL-C, and that pursuing overall lipid subfraction improvements may lead to benefits beyond macrovascular risk reduction for patients with T2DM.

\section{Limitations}

Claims-based studies are subject to important limitations. The coding of claims is susceptible to lack of specificity, miscoding, improper sequencing, and clerical errors. The study may be hindered by incomplete data in terms of insufficient longitudinal capture of relevant claims (e.g. lack of capture of pre-existing MVCs prior to the start of the patient's health plan eligibility) and/or capture of information that is unobservable in an administrative claims database (e.g. use of over-the-counter medications). Unobservable factors that may influence outcomes are generally omitted when using claims data and in the case of this study, the presence of such

Table 4 Sensitivity Analysis: Incidence of MVCs and HDL-C, LDL-C, TG and non-HDL-C Stratified Across LDL-C Goal Attainment

\begin{tabular}{lcccc}
\hline LDL goal attained & \multicolumn{4}{c}{ HR (95 \% C.I) } \\
\cline { 2 - 5 } & HDL goal (yes vs. no) & TG goal (yes vs. no) & HDL + TG goals (yes vs. no) & non-HDL goal (yes vs. no) \\
\hline Yes & $0.804(0.75-0.862)$ & $0.788(0.735-0.845)$ & $0.758(0.705-0.816)$ & $0.785(0.717-0.86)$ \\
No & $0.853(0.802-0.907)$ & $0.785(0.738-0.835)$ & $0.787(0.738-0.84)$ & $0.787(0.711-0.871)$ \\
\hline
\end{tabular}

HR: hazard ratio; Cl: confidence interval; LDL-C: low-density lipoprotein cholesterol; HDL-C: high-density lipoprotein cholesterol; TG: triglycerides. 
unobservable factors are assumed to be occur equally between comparison groups. Important contributory factors of T2DM, such as genetics or alcohol or estrogen use, could not be captured in these data [20]. Because the data used in this study pertain to largely workingage subjects within a managed care population, the findings may not be readily replicable or generalizable to the entire T2DM patient population.

The mean follow-up period in our study was less than 2 years, which may not have been of sufficient duration to determine the onset of MVCs attributable to lipid subfractions. However, because this was an observational claims study, requiring a fixed follow-up period of 4 to 5 years for these patients would have forced survival and subsequently introduced bias. As such, follow-up duration was accounted for as a variable in the survival analysis for risk estimation of MVCs. These inherent study design limitations render the findings hypothesisgenerating and need to be confirmed in a prospective, randomized clinical trial.

\section{Conclusion}

This study demonstrates significant independent associations among HDL-C, TG and non-HDL-C with risk for microvascular events following the diagnosis of T2DM. Attaining ADA goals for non-HDL-C, HDL-C, and TG levels were significantly associated with a reduced risk of MVCs. There was no clear association, however, between the attainment of the ADA goal for LDL-C and microvascular event risk. These findings suggest that to reduce risk for both macrovascular and microvascular events among patients with T2DM, all components of the lipid profile should be treated to ADA-specified target levels. These findings need to be validated in a prospective, randomized clinical trial.

\footnotetext{
Abbreviations

T2DM: Type 2 Diabetes Mellitus; ADA: American Diabetes Association; AHA: American Heart Association; MVC: Microvascular Complication; LDLC: Low-Density Lipoprotein Cholesterol; TG: Triglycerides; HDL-C: HighDensity Lipoprotein Cholesterol; HIRD ${ }^{\text {SM }}$ : HealthCore Integrated Research

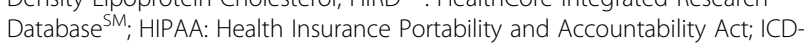
9-CM: International Classification of Diseases Ninth Revision, Clinical Modification; CPT: Current Procedural Terminology; eGFR: Glomerular Filtration Rate; MDRD: Modification of Diet and Renal Disease; CHD: Cardiovascular Heart Disease; NCEP ATP III: National Cholesterol Education Program Adult Treatment Panel III; ACE: Angiotensin-Converting Enzyme; ARB: Angiotensin Receptor Blockers; PDC: Proportion of Days Covered; KM: Kaplan-Meier; RR: Relative Risk; VIF: Inflation Factor; FIELD: Fenofibrate Intervention and Event Lowering in Diabetes; ACCORD: Action to Control Cardiovascular Risk in Diabetes; DAIS: Diabetes Atherosclerosis Intervention Study; HMO: Health Maintenance Organization; PPO: Patient Preferred Organization; POS: Point of Service; FFS: Fee-ForService; DCl: Deyo-Charlson Comorbidity Index; SD: Standard Deviation; Cl: Confidence Interval; HR: Hazard Ratio.
}

\section{Competing interests}

Dr. Toth has disclosed that he serves on the Speakers Bureau for Abbott Laboratories, Amylin, AstraZeneca; GSK, Kowa, and Merck \& Co. He is a consultant for Abbott laboratories, Aegerion, Amgen, Amylin, AstraZeneca
Pharmaceuticals, Kowa, and Merck. The other authors disclosed that they have no competing interests.

\section{Authors' contributions}

PPT led the study, collaborated in its development and design and reviewed the results and manuscript throughout. RJS conceptualized the study, and reviewed and manuscript the results at all stages. DK conceptualized the study, and collaborated with the team on results evaluation and review. RAQ developed the study design and protocol, supervised the statistical analysis and reviewed the results and manuscript throughout. SRP assisted in protocol development, performed statistical analysis, reported and reviewed the results throughout the study and reviewed and edited the manuscript. MJC reviewed the study design and results at various stages of the process. All authors read and approved the final manuscript.

\section{Acknowledgements}

Bernard B. Tulsi, MSc, and Cheryl Jones, BA, provided writing and other editorial support for this manuscript. Ralph A. Quimbo, MA, and Swetha Rao Palli, MS, had full access to the data for the study, and take full responsibility for the integrity of the data and the accuracy of the data analysis.

\section{Author details}

${ }^{1}$ CGH Medical Center, 101 east Miller Rd., Sterling, IL 61081, USA. ${ }^{2}$ University of Illinois College of Medicine, Peoria, IL, USA. ${ }^{3}$ Abbott Laboratories, Abbott Park, IL, USA. ${ }^{4}$ HealthCore, Inc., Wilmington, DE, USA.

Received: 31 May 2012 Accepted: 30 August 2012 Published: 14 September 2012

\section{References}

1. Buse JB, Ginsberg HN, Bakris GL, et al: Primary prevention of cardiovascular diseases in people with diabetes mellitus: a scientific statement from the American Heart Association and the American Diabetes Association. Circulation 2007, 115:114-126.

2. Fioretto P, Dodson PM, Ziegler D, Rosenson RS: Residual microvascular risk in diabetes: unmet needs and future directions. Nat Rev Endocrinol 2010, 6:19-25.

3. Misra A, Kumar S, Kishore VN, Kumar A: The role of lipids in the development of diabetic microvascular complications: implications for therapy. Am J Cardiovasc Drugs 2003, 3:325-338.

4. National Diabetes Statistics 2011 http://www.cdc.gov/diabetes/pubs/pdf/ ndfs_2011.pdf.

5. Rein DB, Zhang $P$, Wirth $K E$, et al: The economic burden of major adult visual disorders in the United States. Arch Ophthalmol 2006, 124:1754-1760.

6. Gordois A, Scuffham P, Shearer A, Oglesby A: The health care costs of diabetic nephropathy in the United States and the United Kingdom. I Diabetes Complications 2004, 18:18-26.

7. Leiter LA: The prevention of diabetic microvascular complications of diabetes: is there a role for lipid lowering? Diabetes Res Clin Pract 2005, 68(Suppl 2):S3-14.

8. Harris SB, Naqshbandi M, Bhattacharyya O, et al: Major gaps in diabetes clinical care among Canada's First Nations: results of the CIRCLE study. Diab Res Clin Pract 2011, 92:272-279.

9. Larsson LI, Alm A, Lithner F, Dahlen G, Bergstrom R: The association of hyperlipidemia with retinopathy in diabetic patients aged $15-50$ years in the county of Umea. Acta Ophthalmol Scand 1999, 77:585-591.

10. Mulec H, Johnsen SA, Wiklund O, Bjorck S: Cholesterol: a renal risk factor in diabetic nephropathy? Am J Kidney Dis 1993, 22:196-201.

11. Forrest KY, Maser RE, Pambianco G, Becker DJ, Orchard TJ: Hypertension as a risk factor for diabetic neuropathy: a prospective study. Diabetes 1997 , 46:665-670.

12. Standards of medical care in diabetes-2010. Diabetes Care 2010, 33(Suppl 1): S11-S61.

13. National Cholesterol Education Program: Third report of the expert panel on detection, evaluation, and treatment of high blood cholesterol in adults (Adult Treatment Panel III). NIH Publication No. 02-5215. Bethesda, MD: National Heart Lung and Blood Institute; 2002.

14. Mosca L, Banka CL, Benjamin EJ, et al: Evidence-based guidelines for cardiovascular disease prevention in women: 2007 update. Circulation 2007, 115:1481-1501. 
15. Leslie RS: Using arrays to calculate medication utilization. SAS Global Forum 2007.

16. Peterson AM, Nau DP, Cramer JA, Benner J, Gwadry-Sridhar F, Nichol M: A checklist for medication compliance and persistence studies using retrospective databases. Value Health 2007, 10:3-12.

17. Costa J, Borges M, David C, Vaz CA: Efficacy of lipid lowering drug treatment for diabetic and non-diabetic patients: meta-analysis of randomised controlled trials. Br Med J 2006, 332:1115-1124.

18. Kordonouri O, Danne T, Hopfenmuller W, Enders I, Hovener G, Weber B: Lipid profiles and blood pressure: are they risk factors for the development of early background retinopathy and incipient nephropathy in children with insulin-dependent diabetes mellitus? Acta Paediatr 1996, 85:43-48.

19. Haffner SM: Management of dyslipidemia in adults with diabetes. Diabetes Care 1998, 21:160-178.

20. Haffner SM: Management of dyslipidemia in adults with diabetes. Diabetes Care 2003, 26(Suppl 1):S83-S86.

21. Zoppini G, Negri C, Stoico V, et al: Triglyceride-high-density lipoprotein cholesterol is associated with microvascular complications in type 2 diabetes mellitus. Metabolism 2012, 61:22-29.

22. Teramoto T, Shirai K, Daida H, Yamada N: Effects of bezafibrate on lipid and glucose metabolism in dyslipidemic patients with diabetes: the JBENEFIT study. Cardiovasc Diabetology 2012, 11:29.

23. Grundy SM, Vega GL, Tomassini JE, Tershakovec AM: Correlation of nonhigh-density lipoprotein cholesterol and low-density lipoprotein cholesterol with apolipoprotein B during simvastatin + fenofibrate therapy in patients with combined hyperlipidemia (a subanalysis of the SAFARI trial). Am J Cardiol 2009, 104:548-553.

24. Lu W, Resnick HE, Jablonski KA, et al: Non-HDL cholesterol as a predictor of cardiovascular disease in type 2 diabetes: the strong heart study. Diabetes Care 2003, 26:16-23.

25. Querton L, Buysschaert M, Hermans MP: Hypertriglyceridemia and residual dyslipidemia in statin-treatment patients with diabetes at the highest risk for cardiovascular disease and achieving very-low low-density lipoprotein-cholesterol levels. J Clin Lipidol. doi:10.1016/j.jacl.2012.04.002.

26. Hermans MP, Ahn SA, Rousseau MF: $\log (\mathrm{TG}) / \mathrm{HDL}-\mathrm{C}$ is related to both residual cardiometabolic risk and $\beta$-cell function loss in type 2 diabetes mellitus. Cardiovasc Diabetology 2010, 9:88.

27. Keech A, Simes RJ, Barter $P$, et al: Effects of long-term fenofibrate therapy on cardiovascular events in 9795 people with type 2 diabetes mellitus (the FIELD study): randomised controlled trial. Lancet 2005, 366:1849-1861.

28. Keech AC, Mitchell P, Summanen PA, et al: Effect of fenofibrate on the need for laser treatment for diabetic retinopathy (FIELD study): a randomised controlled trial. Lancet 2007, 370:1687-1697.

29. Toth PP: Clinical insights from the Fenofibrate Intervention and Event Lowering in Diabetes study: a community practice perspective. Int $J$ Clin Pract 2009, 63:903-911.

30. Chew EY, Ambrosius WT, Davis MD, et al: Effects of medical therapies on retinopathy progression in type 2 diabetes. N Engl J Med 2010, 363:233-244.

31. Ansquer JC, Foucher C, Rattier S, Taskinen MR, Steiner G: Fenofibrate reduces progression to microalbuminuria over 3 years in a placebo-controlled study in type 2 diabetes: results from the Diabetes Atherosclerosis Intervention Study (DAIS). Am J Kidney Dis 2005, 45:485-493.

doi:10.1186/1475-2840-11-109

Cite this article as: Toth et al.: The impact of serum lipids on risk for microangiopathy in patients with type 2 diabetes mellitus. Cardiovascular Diabetology 2012 11:109.

\section{Submit your next manuscript to BioMed Central and take full advantage of:}

- Convenient online submission

- Thorough peer review

- No space constraints or color figure charges

- Immediate publication on acceptance

- Inclusion in PubMed, CAS, Scopus and Google Scholar

- Research which is freely available for redistribution

Submit your manuscript at www.biomedcentral.com/submit
Ciomed Central 